\title{
Development of Mathematical Models to Estimate Optimum Capacity Assignment Network Connections
}

\author{
Khaled Batiha \\ Prince Hussein Bin Abdullah \\ Faculty of Information Technology, \\ Al al-Bayt University, Jordan
}

\begin{abstract}
In this paper, the optimal characteristics of network structures are considered. The mathematical model to estimate the optimum bandwidth of communication networks for the arbitrary law of the length distribution of a transmitted message founded.
\end{abstract}

\section{General Terms}

Network, Mathematical models

\section{Keywords}

Queuing theory, probability theory, stochastic processes, theory of mathematical modeling, network design theory, theory of mathematical programming.

\section{INTRODUCTION}

Designing an efficient topology and flow assignment strategy that could meet the requirements at the lowest costs was essential to outperform the competition represented by networks. The challenge was to solve a combined flow and capacity assignment problem.

The main difficulty in the widespread introduction of a data communication system (DCS) distributed in sectors of the economy is the overly high cost. Therefore, finding opportunities to reduce the costs incurred by the network and improve the efficiency of the use of material and human resources of the existing DCS is an urgent problem, part of which solved not good enough.

The main problem with research on designing a highly affective DCS is to determine the technical requirements for the basic characteristics and parameters of the network and its components based on solving the problem of choosing the optimal resource and flow control, among other issues. The most difficult problem with creating DCSs is selecting or creating the optimal structure of the network and defining the basic material costs of the network. To solve this problem, mathematical models are developed, which are the basic tools in decision-making regarding the creation of DCS structures, based on which a method of a system design of optimal networks can be developed.

Regarding the importance of the practical and theoretical work on the existing DCS, it should be noted that many of the results are based on the private criteria of efficiency (performance, reliability, noise immunity, cost, etc.), which are considered to have no relationship with each other.

Therefore, an urgent task of scientific research is to evaluate the effect of DCS distribution laws on property values and DCS channels. In discussing the laws of the distribution of random variables, a DCS cannot disregard the fact that the transmission of information in real networks is based on protocols developed by the International Organization for Standardization. Protocols define the procedures for connection management, the sequence data of various service teams in the network, and the format of the messages and other processes in exchanging data. It is obvious that the protocols are leading to significant changes to the distribution of transmitted messages. Therefore, one of the main objectives of this study should be conducting impact assessment reports on the value of the optimal characteristics of DCS structures.

To achieve this goal, it is necessary to develop mathematical models to estimate the optimum bandwidth of communication networks for the arbitrary law of the length distribution of transmitted messages.

\section{RELATED WORKS}

We shall outline several approaches to the capacity assignment utilizing different performance criteria. Much of this is based on the pioneering work of Kleinrock [5]. In the first (and simplest) case, due specifically to Kleinrock, we assume cost is linearly proportional to capacity.

In the capacity assignment discussion in Schwartz [7], he emphasized a technique in which the average message time delay is minimized, while the total capacity is fixed. A variation of this, leading to essentially the same results.

Prior to joining UCLA, Kleinrock had already formulated and solved the capacity assignment problem in his $1962 \mathrm{PhD}$ dissertation, which was later republished as a book [5] in 1964.

Practical simplifications have been found in the literature since the pioneering work of Fratta et al. [3] by minimizing the average delay measure on all active arcs.

Many studies [4] review the basic flow deviation (FD) procedure of assigning flows within store-and-forward communication networks to minimize costs and/or delay a given topology, and the given external flow requirements and various approaches to the problem are discussed, and the FD method is introduced and described.

For our ongoing research, of particular interest are the studies of Kleinrock [5], [6] and Martin [7]. Kleinrock [5], [6] considered the problem of determining the bandwidth of communication channels and the flow distribution in networks. He examined the different values depending on the bandwidth of the channel, which was very interesting and useful for the design formula. However, these formulas are valid only under the condition that the communication channels are reliable and the circulating flow in the network is the same length as the messages. This limits their ability to calculate circuits as determined in the works of Kleinrock [5], [6] as other researchers can only serve as estimates. However, his works are studied with the same network for a long stream of messages. For our ongoing research, to merit attention that deals with the task of building a communication network of a minimum length, there are examples of the calculation of 
network structures. Of course, this material should be used in the task of constructing an optimal structure in terms of the choice of location on the ground.

To add value to the practice of network design, the work of Schwartz [9] will be referenced. Kleinrock and Schwartz in their works [5], [6] [9] give much attention to the design of the network structure, while considering the advantages and disadvantages of the already developed algorithms to optimize structures like Prim algorithm, Kruskal's algorithm et al.. However, in this work, it is assumed that the network is transmitted on homogeneous Poisson message arrivals and that channels are reliable.

It should also be noted that in the works selected for solving network design issues, the effect of the characteristics of the structure of the used protocol is not examined. Therefore, questions of influence, especially regarding the risks, should be the basis for our ongoing research.

Zaichenko in his work [10] design the structure of networks, he uses the results of the graph theory, as the weights of the edges, which rely on the known functional dependence of the value of the communication channels regarding the length of the bandwidth, which is not always known. In addition, they suppose that the flow on the network is homogeneous. When calculating the known structure of the network connectivity, it is believed to be a separate problem, the solution of which will depend on the structure of the network.

Designing the optimal structure of the network is closely related to the synthesis of a topological network. Topological syntheses are solved with the help of the graph theory. Among the many works on this theory, its application to the analysis of the synthesis of networks should be allocated [Frank, 1971]. In these studies is a detailed description of almost all modern methods of research networks.

In a 2009, [8] Reaz optimized cost performance tradeoffs, suggesting an access network that uses a combination of optical and radio links. In a 2011 paper, Chiaraviglio [1] solved the problems of energy consumption and greenhouse effects. The authors educe the energy budget through the optimization of energy generation, transportation, and consumption, proposed the use of information and communication technologies (ICT).

In a 2009 Reaz in his paper [8] minimized the delay and increased the throughput in the wireless mesh network (WMN) of a wireless-optical broadband access network (WOBAN) by designing a capacity- and delay-aware routing scheme, called CaDAR. In another paper, Chiaraviglio [1] solved the problem of minimizing power consumption for Internet service provider (ISP) networks.

In conclusion, it should be noted that the above list of literature works, as well as others not mentioned here, which are studies on network design that posed questions, by minimizing the cost of the network are solved without the necessary network connectivity, which does not allow assessing the optimality of solved problem.

Based on the analysis model, determining the optimal network structure shown in the literature, it was found that the existing models are built on the assumption that does not always correspond to the real operating conditions of networks. Thus, the mathematical models do not take into account the characteristics of the transmission of information, as defined by the recommendations of the International Advisory Committee for telephony and telegraphy, which refer to the procedures for packet-level services, a virtual connections set of service commands, and others. These features deny the assumption of the exponential distribution of the lengths of the transmitted messages.

\section{FORMULATION OF THE PROBLEM}

When the statement of the problem is formulated then we must select the network topology and throughput of communication channels in which provided the following conditions:

$$
\begin{aligned}
& \sum_{\mathrm{i}=1}^{\mathrm{N}} \mathrm{d}_{\mathrm{i}} \mathrm{C}_{\mathrm{i}}=\min \\
& \sum_{\mathrm{i}=1}^{\mathrm{N}} \frac{\lambda_{\mathrm{i}}}{\gamma} \overline{\mathrm{T}}_{\mathrm{i}} \leq \mathrm{T}_{\mathrm{gc} 3},
\end{aligned}
$$

$\mathrm{T}_{\mathrm{gc} 3}$ - Defined by the customer or user of the average message delivery time (or a package) in the network,

$d_{i}-$ The cost incurred for each unit of capacity built into the ith channel.

$\mathrm{C}_{\mathrm{i}}$-The channel capacity of the ith channel (bps),

$\overline{\mathrm{T}}_{\mathrm{i}}$-The average time delay for messages in the network

$\lambda_{\mathrm{i}}$ - The ith link message rate (message / sec)

$\gamma$ - The total external message arrival rate

Here, as before, the network topology is determined by the model of the graph theory; however, the main problem is defining the depending $\bar{T}_{i}$ based on the characteristics of the different flows circulating in the network, the capacity assignment, the reliability of communication channels, and other factors affecting the efficiency of the network. Having such a relationship and knowing the limitations imposed on the time delivery of messages $\mathrm{T}_{\mathrm{gc} 3}$, providing conditions (1) and (2) the desired link capacity assignment can be found, as well as its required reliability, and other characteristics of the network structure. This is the essence of an integrated approach to solving the problem of the choice of a rational structure for the network.

Introducing the single-channel queuing system, which receives a number of different Poisson streams differing in the average value of the message length is exponentially distributed, it was obtained by the following estimated expression:

$$
\mathrm{T}_{\mathrm{i}}=\frac{\sum_{\mathrm{l}=1}^{\mathrm{k}} \rho_{\mathrm{l}} \overline{\mathrm{\tau}}_{\mathrm{l}}}{1-\sum_{\mathrm{l}=1}^{\mathrm{k}} \rho_{\mathrm{l}}}+\frac{\rho_{\mathrm{i}}}{\lambda_{\mathrm{i}}}
$$

where

$\rho_{1}=\lambda_{1} \bar{\tau}_{1}=\frac{\lambda_{i}}{\mu_{1}}$ - loading ith channel lth message flow, the average length, which equals $\overline{\tau_{l}}(\mathrm{c})$;

$\mu_{1}$ - The intensity of the message service lth stream;

$\rho_{\mathrm{i}}=\sum_{\mathrm{l}=1}^{\mathrm{k}} \rho_{\mathrm{l}}-$ Total loading all the ith channel stream of messages;

$\mathrm{K}$ - The amount of different (non-uniform) message flows;

$\lambda_{\mathrm{i}}=\sum_{\mathrm{l}=1}^{\mathrm{k}} \lambda_{\mathrm{l}}$ - The intensity of the total flow in the ith link. 
In (3), the first term is the average wait time messaging in the ith channel, and the second is the average service time of messages to the stream.

As discussed regarding earlier models, Kleinrock and others have not considered the heterogeneity of flows, but it used the average value of the average service time $T$ for all to the flow circulating in the ith channel.

The problem of evaluating the optimum capacity assignment of communication channels among the most complex structures is in the synthesis of the distribution of the network. The difference that is considered for assessing the capacity of channels, which is given in the previous section, is that it is based on many inhomogeneous streams transmitted over the communication channel. It is necessary to minimize the costs of the communication channels defined by their capacity. A comprehensive approach to solving the problem of building a network statement of the problem, as already noted, is as follows.

Known features of inhomogeneous Poisson streams in each channel of communication, i.e. known $\left\{\lambda_{\mathrm{li}}, \mu_{\mathrm{li}}\right\},(\mathrm{l}=\overline{1, \mathrm{k}}, \mathrm{i}=$ $1, \mathrm{~N}$,

Vary: Channel capacity.

Restriction: The average delay time for each pair of subscribers connected to the ith channel should not exceed the predetermined value for the channel $\mathrm{T}_{\mathrm{gc} 3}$

Minimize: capacity assignment distributed network.

To solve this problem, we used formula (3) with the following remarks. The transmission of data on channels is performed using messages or packets whose lengths are measured in bits. In formula (3), message lengths are measured in seconds.

This length depends on the transmission rate data, i.e., the capacity assignment of $C_{i}$. In general, this dependence is expressed by the ratio:

$$
\bar{\tau}_{i}(s)=\frac{\bar{\tau}_{i}^{(b)}}{C_{i}},
$$

$\bar{\tau}_{i}{ }^{(s)}$ - The average length of the message (packet) in seconds.

$\bar{\tau}_{i}{ }^{(b)}$ - The average length of the message (packet) in bits

$\mathrm{C}_{\mathrm{i}}$ - The capacity assignment connection.

To determine the optimal LINK CAPACITY ASSIGNMENT, we must substitute (4) in (3) and equate its value as specified on the restriction of the channelT $\mathrm{T}_{\mathrm{gc} 3 \mathrm{i}}$. Then, we can write:

$$
\mathrm{T}_{\mathrm{gc} 3 \mathrm{i}}=\frac{\sum_{\mathrm{l}=1}^{\mathrm{k}} \lambda_{\mathrm{l}}\left(\overline{\mathrm{\tau}}_{\mathrm{i}}{ }^{\left.(\mathrm{b})^{2}\right)}\right.}{\mathrm{C}_{\mathrm{i}}\left(\mathrm{C}_{\mathrm{i}}-\sum_{\mathrm{l}=1}^{\mathrm{k}} \lambda_{\mathrm{l}} \overline{\mathrm{\tau}}_{\mathrm{l}}^{(\mathrm{b})}\right)}+\frac{\rho_{\mathrm{i}}^{(\mathrm{b})}}{\mathrm{C}_{\mathrm{i}} \lambda_{\mathrm{i}}},
$$

Where $\rho_{\mathrm{i}}^{(\mathrm{b})}=\sum_{\mathrm{l}=1}^{\mathrm{k}} \lambda_{\mathrm{l}} \bar{\tau}_{\mathrm{l}}{ }^{(\mathrm{b})}$ - total load of the ith channel-uniform flow, as measured in bits/sec. The remaining symbols correspond to notation (3).

The solution to (5) with respect to $C_{i}$ is it yields the final expression for determining an optimum capacity assignment of the ith channel network, which has the form:

$$
\mathrm{C}_{\mathrm{i}}^{\text {opt. }}=\frac{\mathrm{b}+\sqrt{\mathrm{b}^{2}+4 \cdot \mathrm{S} \cdot \mathrm{n}}}{2 \mathrm{~S}}
$$

$$
\begin{gathered}
\text { Where } \mathrm{b}=\left(\lambda_{\mathrm{i}} \cdot \mathrm{T}_{\mathrm{gc} 3 \mathrm{i}}+1\right) \cdot \sum_{\mathrm{l}=1}^{\mathrm{k}} \lambda_{\mathrm{l}} \overline{\mathrm{\tau}}_{\mathrm{l}}{ }^{(\mathrm{b})}, \mathrm{S}= \\
\lambda_{\mathrm{i}} \cdot \mathrm{T}_{\mathrm{gc} 3 \mathrm{i}}, \\
\mathrm{n}=\lambda_{\mathrm{i}} \cdot \sum_{\mathrm{l}=1}^{\mathrm{k}} \lambda_{\mathrm{l}}\left(\bar{\tau}_{\mathrm{l}}{ }^{(\mathrm{b})}\right)^{2}-\left(\sum_{\mathrm{l}=1}^{\mathrm{k}} \lambda_{\mathrm{l}} \overline{\mathrm{\tau}}_{\mathrm{l}}{ }^{(\mathrm{b})}\right)^{2}
\end{gathered}
$$

Expression (6) represents the minimum required capacity assignment and, consequently, its minimum value depending on the capacity at which the following condition is satisfied $\overline{\mathrm{T}}_{\mathrm{gci}}=\mathrm{T}_{\mathrm{gc} 3 \mathrm{i}}-$ this expression is appropriate to apply to the calculation of capacities of channels, as the network structure of the reliability corresponds to the desired ratio of readiness.

\section{CONCLUSION}

The main result is to develop mathematical models to calculate the bandwidth of communication, which in contrast to the known laws considers the effect of the length distribution of messages to be transmitted to the optimum value of the network bandwidth that is needed to assess the optimal structure of the network. Mathematical models brought to the settlement dependence are convenient in practicing network design.

\section{REFERENCES}

[1] L. Chiaraviglio, M. Mellia, F. Neri, 2012 Minimizing ISP network energy cost: formulation and solutions, IEEE/ACM Trans Netw (TON) 20 (2) 463-476.

[2] H. Frank, T. Frish, 1971 Communication, Transmission, and Transportation Networks, Addison- Wesley, Reading, Mass..

[3] L. Fratta, M. Gerla, L. Kleinrock, 1973 The flow deviation method: an approach to store-and-forward communication network design, Networks 3 (2) 97-133.

[4] L. Fratta, M. Gerla, L. Kleinrock, 2014 Flow Deviation: 40 years of incremental flows for packets, waves, cars and tunnels, Computer Networks 66,18-31

[5] L. Kleinrock, 2007 Communication Nets: Stochastic Message Flow and Delay, McGraw-Hill Book Company, 1964 (Out of Print) Reprinted by Dover Publications, 1972 and in 2007.

[6] L. Kleinrock, 1970 Analytic and simulation methods in computer network design, in: Proceedings of the Spring Joint Computer Conference, Atlantic City, New Jersey, May 5-7, 569-579.

[7] J. Martin, 1972 Systems Analysis for Data Transmission, Prentice-Hall, Englewood Cliffs, N.J.

[8] A.S. Reaz, V. Ramamurthi, S. Sarkar, D. Ghosal, S. Dixit, B. Mukherjee, 2009 CaDAR: an efficient routing algorithm for a wireless-optical broadband access network (WOBAN), J Opt Commun Netw 1 (5) 392 403 .

[9] M. Schwartz, 1977 Computer- Communication Network Design and Analysis, Prentice-Hall, Englewood Cliffs, N.J.

[10] Y. P. Zaichenko, 2003 Operations Research, Publishing House "Word", Kiev. 